\title{
O relaxamento respiratório no manejo do craving e dos sintomas de ansiedade em dependentes de crack
}

\author{
Deep breathing in the management of craving and anxiety symptoms of crack-cocaine- \\ dependent patients
}

\author{
Taís Cardoso de Zeni' ${ }^{1}$, Renata Brasil Araujo \\ ${ }^{1}$ Educadora física. Residente, Saúde Mental Coletiva, Escola de Saúde Pública, Porto Alegre, RS. ${ }^{2}$ Psicóloga. Doutora, Psicologia. Hospital Psiquiátrico São Pedro, Porto \\ Alegre, RS. \\ O presente estudo é uma comunicação breve, resultado de uma pesquisa apresentada do trabalho de conclusão de curso da Residência Integrada em Saúde Mental Coletiva, \\ Escola de Saúde Pública, Porto Alegre, RS, apresentado e avaliado por banca científica no dia 09/01/09 na mesma instituição.
}

\section{Resumo}

Introdução: O objetivo deste estudo foi verificar a efetividade do relaxamento respiratório no manejo do craving e dos sintomas de ansiedade em dependentes de crack internados para tratamento em uma unidade de desintoxicação.

Método: Ensaio clínico do tipo quase-experimental de análise quantitativa. A amostra foi por conveniência, sendo composta por 32 homens dependentes de cocaína ( $\mathrm{rack}$ ). Eles tinham a cocaína como a droga de escolha e haviam utilizado esta substância por última vez entre 2 e 3 semanas antes do início do tratamento, conseguindo realizar a técnica do relaxamento respiratório adequadamente do ponto de vista biomecânico. Os instrumentos aplicados foram: Cocaine Craving Questionnaire-Brief (CCQB), escala analógica visual (EAV), Inventário Beck de ansiedade (BAI) e ficha com dados sociodemográficos e referentes ao padrão de consumo de substâncias psicoativas (FSD). Foi realizada uma intervenção em grupo na qual, inicialmente, foram aplicados o CCQB, a EAV e o BAI. Depois, foram apresentadas imagens relacionadas ao uso do crack e foram reaplicados os mesmos instrumentos. A seguir, foi realizado o relaxamento respiratório durante 10 minutos e foram aplicados, pela terceira vez, os instrumentos. Após esta intervenção, foi realizada uma entrevista individual com aplicação da FSD.

Resultados: Os resultados desta pesquisa demonstraram uma redução dos escores do CCQB, da EAV e do BAI pelo relaxamento respiratório em uma amostra cujo perfil corresponde ao padrão geral dos usuários de crack.

Conclusão: Este estudo, apesar de ter algumas limitações metodológicas, sugere que o relaxamento respiratório pode ser uma estratégia efetiva no manejo do craving e dos sintomas de ansiedade em dependentes de crack.

Descritores: Cocaína crack, síndrome de abstinência a substâncias, ansiedade.

\begin{abstract}
Introduction: The objective of this study was to verify the effectiveness of deep breathing in the management of craving and anxiety symptoms in crack-cocaine-dependent patients hospitalized for treatment in a detoxification unit.

Method: This is a quasi-experimental clinical trial using a quantitative analysis. The convenience sample comprised 32 crack-cocaine-dependent males. These subjects had cocaine as their drug of choice, having used this substance between 2 and 3 weeks prior to the beginning of the treatment, and were able to adequately perform the deep breathing technique. The instruments used were: the Cocaine Craving Questionnaire-Brief (CCQB), the visual analogue scale (VAS), the Beck Anxiety Inventory (BAI), and a form containing sociodemographic information and data related to the pattern of consumption of psychoactive substances (SDF). A group intervention was conducted with the administration of the CCQB, the VAS, and the BAI. After that, images related to the use of crack-cocaine were shown to the subjects and the same instruments were administered again. Next, the participants performed the deep breathing technique for 10 minutes, and the instruments were administered one more time. Finally, an individual interview was done and the SDF was completed.

Results: Our findings demonstrated a decrease in the scores on the CCQB, the VAS and the BAI after the deep breathing technique was performed in a sample whose profile represents the general pattern of crack-cocaine users.

Conclusion: In spite of some methodological limitations, the present study suggests that the deep breathing technique is effective in the management of craving and anxiety symptoms in crack-cocaine-dependent patients.
\end{abstract}

Keywords: Crack-cocaine, substance withdrawal syndrome, anxiety.

Correspondência:

Taís Cardoso de Zeni, Rua Ferreira Viana, 649/203, CEP 90670-100, Bairro Petrópolis, Porto Alegre, RS. Tel.: (51) 8133.8527. E-mail: taiszeni@hotmail.com

Não há conflitos de interesse associados à publicação deste artigo.

Copyright (C) Revista de Psiquiatria do Rio Grande do Sul - APRS

Recebido em 21/09/2008. Aceito em 19/05/2009. 


\section{Introdução}

A dificuldade para interromper o uso de qualquer droga está relacionada com a falta de habilidade para resistir ao impulso de utilizá-la ao enfrentar o craving. O craving, entendido como um desejo súbito e intenso de usar uma determinada substância ${ }^{1}$, é uma experiência idiossincrática formada por componentes cognitivos, afetivos, comportamentais e fisiológicos. Esse desejo irresistível, por vezes inconsciente, e que se sobrepõe, frequentemente, à vontade convicta da manutenção da abstinência, pode levar o dependente à recaída e, inclusive, ao abandono terapêutico, mesmo depois de grandes períodos de abstinência ${ }^{2}$.

O crack é o cloridrato de cocaína processado com outras substâncias em forma de "pedras" que podem ser fumadas. Após a cachimbada, o início da ação da cocaína (high) acontece quase instantaneamente - em 5 a 10 segundos $^{3}-$, estimulando o sistema nervoso central, havendo o bloqueio da recaptação de dopamina, serotonina e noradrenalina nas sinapses. No entanto, esses efeitos estimulantes se dissipam rapidamente - em 5 a 10 minutos -, e o usuário passa à intensa depressão do sistema nervoso central; nesse momento, dominado pela ansiedade e pelo craving, é levado à compulsão por uma nova dose. O potencial de abuso e de dependência é bem mais elevado na via pulmonar de administração da cocaína do que em outras vias (oral, nasal, intravenosa), pois a ação da droga é mais intensa e, ao mesmo tempo, possui uma reduzida duração $0^{3,4}$.

No tratamento da dependência de cocaína, principalmente na redução do craving, não há consenso nem resultados promissores quanto à eficácia da psicofarmacologia ${ }^{5}$, o que torna essencial uma abordagem multidisciplinar e integral do usuário, com a utilização de técnicas cognitivo-comportamentais que enfoquem, principalmente, as estratégias de prevenção de recaída ${ }^{6}$. O craving é considerado uma variável importante a ser observada no tratamento do dependente da cocaína e é fundamental reforçar a habilidade do usuário da droga para experimentar este fenômeno sem recair ${ }^{3}$. É necessário que o dependente aprenda a identificar e enfrentar o craving, assim como os sinais e os estímulos associados ao mesmo, no sentido de criar a habilidade de resistir a esse desejo. Em sessões terapêuticas, o craving pode ser primeiramente induzido e posteriormente reduzido através de técnicas de enfrentamento ${ }^{2}$.

No manejo do craving, uma das técnicas de enfrentamento utilizada na terapia cognitivo-comportamental é o relaxamento respiratório (RR). Um estudo realizado com alcoolistas sugere o relaxamento como estratégia efetiva para controlar os sintomas de ansiedade diante do craving $^{7}$. Outro estudo, com tabagistas, afirma que a respiração profunda pode reduzir significativamente o craving e aliviar outros sintomas da abstinência do tabaco ${ }^{8}$.

O objetivo deste estudo foi verificar a efetividade do RR no manejo do craving e dos sintomas de ansiedade nos dependentes de crack internados para tratamento em uma unidade de desintoxicação.

\section{Método}

Este estudo caracteriza-se como um ensaio clínico do tipo quase-experimental de análise quantitativa. O projeto foi aprovado pelo Comitê de Ética do Hospital Psiquiátrico São Pedro (HPSP), Porto Alegre (RS). A assinatura do termo de consentimento livre e esclarecido foi considerada précondição para a participação nesta pesquisa. Os dados foram coletados de maio a outubro de 2008 na unidade de desintoxicação masculina do HPSP, em 9 sessões de intervenção psicoeducativa em grupo e em entrevistas individuais junto à amostra selecionada. Os dados coletados foram analisados no programa Statistical Package for the Social Sciences (SPSS), versão 12.0. Os dados foram tratados estatisticamente, sendo feita uma análise descritiva e de frequência dos dados sociodemográficos e referentes ao padrão de consumo de substâncias psicoativas. Foi utilizado o teste $t$ de Student para amostras pareadas para o estudo inferencial. O nível de significância foi de $5 \%$.

Os instrumentos utilizados foram: Inventário Beck de ansiedade (Beck Anxiety Inventory, BAI) ${ }^{9,10}$, para avaliar sintomas de ansiedade; escala analógica visual (EAV) ${ }^{11}$ e Cocaine Craving Questionnaire-Brief (CCQB) ${ }^{12}$, para avaliar a presença do craving por cocaína; e ficha de dados sociodemográficos (FSD) e referentes ao padrão de consumo de substâncias psicoativas, para identificar características gerais da amostra e fatores relacionados ao consumo de drogas.

A intervenção foi realizada em uma sala fechada com poucas interferências externas. O grupo foi constituído por de 3 a 6 participantes por adesão voluntária. Os participantes sentaram em cadeiras dispostas em círculo em torno de uma mesa. Inicialmente foram avaliados os sintomas de ansiedade e o craving por cocaína através dos instrumentos BAI, EAV, CCQB. Depois, foram expostas durante 1 minuto oito imagens fotográficas relativas ao crack e ao seu uso ("pedras", cachimbos, latas). Em seguida, foram reaplicados os instrumentos BAI, EAV, CCQB, a fim de avaliar a ocorrência de indução do craving e dos sintomas de ansiedade. Depois, os participantes foram conduzidos verbalmente a realizar a técnica do RR durante 10 minutos, ao som de uma música relaxante, na posição sentada, com os olhos fechados e as palmas das mãos apoiadas sobre a região abdominal.

Do ponto de vista biomecânico, o RR é uma técnica que consiste no exercício de controle da respiração através da musculatura diafragmática, realizada em três fases distintas:

1. Inspirar pelas narinas, distendendo o diafragma, dilatando o abdome e contando 3 tempos;

2. Prender o ar nos pulmões, contando 3 tempos;

3. Expirar pela boca suavemente, encolhendo o diafragma e contraindo o abdome, contando 6 tempos.

Depois da realização do $\mathrm{RR}$, foram aplicados pela terceira vez o BAI, a EAV e o CCQB para avaliar a ocorrência de redução do craving e dos sintomas da ansiedade. Concluída a intervenção, esclareceu-se que o relaxamento provoca sensação de bem-estar; e que, em estado de relaxamento, é 
menos provável que uma pessoa atue impulsivamente ${ }^{2}$; sendo também salientado que o aprendizado desta técnica pode ser útil aos dependentes de crack, no manejo do craving e dos sintomas de ansiedade.

No intuito de delinear o perfil da amostra selecionada e avaliar se os sujeitos preenchiam mais algum critério de exclusão, foi realizada uma entrevista individual - na qual foi aplicada a FSD - com aqueles participantes que conseguiram realizar a técnica do RR adequadamente do ponto de vista biomecânico.

A amostra foi por conveniência e composta por 32 homens com diagnóstico de dependência de cocaína ( $\mathrm{crack}$ ) pela CID-10 ${ }^{13}$. Eles tinham esta como a droga de escolha e tinham usado esta substância pela última vez de 2 a 3 semanas antes do início do tratamento, realizando a técnica do RR adequadamente na sessão, do ponto de vista biomecânico. Foram excluídos desta amostra sujeitos com hipótese diagnóstica de retardo mental e/ou que apresentavam sintomas psicóticos.

\section{Resultados}

A amostra deste estudo foi composta por homens com média de idade de 24,63 anos (desvio padrão $=4,64$; 18-40), procedentes, na sua maioria $(72,7 \%)$, da região metropolitana de Porto Alegre. A renda mensal média declarada foi de R $\$ 606,56$ (desvio padrão $=313,04 ; 0-1.200,00$ ), aproximadamente 1,26 salários-mínimos. Estes dependentes de crack (100\%) eram também dependentes de tabaco (100\%), de maconha $(93,9 \%)$, de álcool $(57,6 \%)$ e de solventes $(24,2 \%)$. Quanto ao uso do crack, consumiam em média 67,73 pedras por semana, o que daria em torno de 271 pedras por mês. A Tabela 1 demonstra as alterações, segundo o teste $t$ de Student para amostras pareadas, nas médias do craving antes e depois da apresentação de imagens do crack e nas médias do craving e ansiedade antes e depois do RR.

\section{Discussão}

A amostra desta pesquisa demonstra a predominância do consumo do crack em adultos jovens, de baixa renda, usuários de múltiplas drogas ou com histórico de consumo de outras substâncias psicoativas - especialmente álcool, tabaco e maconha -, demonstrativo condizente com outros estudos referentes ao perfil dos usuários de rrack $^{14-17}$.

$\mathrm{Na}$ intervenção psicoeducativa em grupo, houve significativa indução do craving através dos escores do CCQB e da EAV a partir da exposição de imagens relativas ao uso de crack. Alguns participantes negaram a indução do craving pelas imagens, fato que pode ter ocorrido tanto pela dificuldade de reconhecer em si o craving, como pelo medo de relatar o craving e ter sua alta adiada. Outros relataram que não experimentaram o craving através das imagens, pois estavam em ambiente protegido, continente e afastado do acesso

Tabela 1 - Comparação das médias e desvios padrão de craving e ansiedade antes e depois da apresentação de imagens do crack e do craving e ansiedade antes e depois do RR $(\mathrm{n}=32)$

\begin{tabular}{|c|c|c|c|c|}
\hline Variáveis & Média & Desvio padrão & $\mathbf{T}$ & $\mathbf{p}$ \\
\hline EAV & & & $-6,27$ & $<0,001$ \\
\hline EAV antes das imagens do crack & 1,28 & 1,67 & & \\
\hline EAV depois das imagens do crack & 3,53 & 2,84 & & \\
\hline CCQB & & & $-3,71$ & 0,001 \\
\hline CCQB antes das imagens do crack & 19,03 & 5,43 & & \\
\hline CCQB depois das imagens do crack & 25,06 & 10,86 & & \\
\hline BAI & & & $-5,85$ & $<0,001$ \\
\hline BAI antes das imagens do crack & 6,75 & 5,41 & & \\
\hline BAI depois das imagens do crack & 12,84 & 7,76 & & \\
\hline EAV & & & 6,32 & $<0,001$ \\
\hline EAV antes do RR & 3,53 & 2,84 & & \\
\hline EAV depois do RR & 1,22 & 1,82 & & \\
\hline CCQB & & & 4,55 & $<0,001$ \\
\hline CCQB antes do RR & 25,06 & 10,86 & & \\
\hline CCQB depois do RR & 17,00 & 2,27 & & \\
\hline BAI & & & 6,18 & $<0,001$ \\
\hline BAI antes do RR & 12,84 & 7,76 & & \\
\hline BAI depois do RR & 4,34 & 4,05 & & \\
\hline
\end{tabular}

$\mathrm{BAI}=$ Inventário Beck de ansiedade; $\mathrm{CCQB}=$ Cocaine Craving Questionnaire Brief; $\mathrm{EAV}=$ escala analógica visual; $\mathrm{RR}=$ relaxamento respiratório. 
à droga. Alguns relataram que a indução do craving se deu mais pelas falas e pelo comportamento dos participantes, que se alteraram no momento da apresentação das imagens, quando foram observados gírias e trejeitos peculiares.

Após a realização da técnica do $R R$, houve diminuição dos sintomas de ansiedade, observados através do BAI, e uma significativa redução do craving, medida através dos CCQB e EAV. Estes resultados demonstram uma sobreposição dos sintomas de ansiedade e do craving, sugerindo a existência de mecanismos comuns subjacentes a ambos, conforme achados de estudos já realizados com dependentes químicos ${ }^{7,18}$, e a manifestação conjunta dos sintomas de hiperventilação, ansiedade e craving $^{19}$. Como o padrão respiratório de quem está ansioso tende à hiperventilação, que, por sua vez, conduz aos sintomas fisiológicos decorrentes de um aumento significativo de oxigenação sanguínea - tontura, parestesias, sufocação e taquicardia ${ }^{20}-$, o RR pode ser uma potente técnica para o controle de ansiedade, pois pode gerar uma sensação de autocontrole, reduzindo a vulnerabilidade para a hiperventilação ${ }^{21}$. Apesar de os mecanismos de redução do craving e os sintomas de ansiedade não serem amplamente conhecidos, o RR pode ser uma estratégia recomendada aos dependentes de crack para proporcionar redução do craving, graças aos efeitos ansiolíticos inerentes ao RR e à reprodução parcial das características do uso do crack: ao mimetizar a ação do ato de fumar através da respiração diafragmática controlada, o RR induz uma experiência sensório-motora que alivia o craving $^{8}$.

A intervenção realizada neste estudo proporcionou a experiência da técnica do RR como uma estratégia de prevenção de recaída que pode ser ensinada no ambiente clínico e ser aplicada em qualquer momento e lugar. Se convertido em hábito e realizado adequadamente, o RR é uma técnica que pode contribuir com o aumento do senso de autoeficácia do dependente de crack, pois pode melhorar a sua capacidade de lidar com a ansiedade e o craving - fatores centrais do comportamento abusivo ${ }^{7}$.

Esta pesquisa apresenta algumas limitações metodológicas que impossibilitam atribuir a redução do craving e dos sintomas de ansiedade somente à realização da técnica do RR. $\mathrm{O}$ uso da música relaxante e o próprio tempo decorrido da realização do RR (10 minutos) podem ter influenciado na diminuição dos escores das variáveis. Para comprovar, de fato, a eficácia do RR no manejo do craving e dos sintomas de ansiedade, seria necessário realizar um estudo com uma amostra maior, um tempo menor de realização do RR (5 minutos) e com comparação dos escores com grupo controle. Também, seria pertinente verificar a aplicação da técnica fora do ambiente protegido da internação, ou seja, diante de situações de risco do cotidiano dos dependentes de crack, a fim de verificar o seguimento dos efeitos terapêuticos do RR.

\section{Referências}

1. Araujo RB, Oliveira MS, Moraes JF, Pedroso RS, Port F, de Castro MG. Validation of the Brazilian version of Questionnaire of Smoking Urges-Brief. Rev Psiquiatr Clin. 2007;34(4):166-75.

2. Beck A, Newman C, Wright F. Terapia cognitiva de las drogodependencias. Barcelona: Ediciones Paidos Iberica; 2000.

3. Seibel SD, Toscano Jr A. Dependência de drogas. São Paulo: Atheneu; 2001.

4. World Health Organization. Neuroscience of psychoactive substance use and dependence. Geneva: WHO; 2004

5. Focchi GR, Leite MC, Scivoletto S. Utilização do agonista dopaminérgico pergolida no tratamento da "fissura" por cocaína. Rev Bras Psiquiatr. 2005;23(4):188-94.

6. Marlatt AG, Gordon JR. Prevenção de recaída. Porto Alegre: Artes Médicas; 1993.

7. Almeida SC, Araújo RB. Avaliação da efetividade do relaxamento na variação dos sintomas da ansiedade e da fissura em pacientes em tratamento de alcoolismo. Bol Saude. 2005;19(2):135-42.

8. McClernon FJ, Westman EC, Rose JE. The effects of controlled deep breathing on smoking withdrawal symptoms in dependent smokers. Addict Behav. 2004;29(4):765-72.

9. Beck AT, Steer RA. Beck anxiety inventory manual. San Antonio: Psychological Corporation; 1993.

10. Cunha JA. Manual da versão em português das escalas Beck. São Paulo: Casa do Psicólogo; 2001.

11. Dols M, van den Hout M, Kindt M, Willems B. The urge to smoke depends on the expectation of smoking. Addiction. 2002;97(1):87-93.

12. Sussner BD, Smelson DA, Rodrigues S, Kline A, Losonczy M, Ziedonis D. The validity and reliability of a brief measure of cocaine craving. Drug Alcohol Depend. 2006;83(3):233-7.

13. Organização Mundial da Saúde. Classificação de transtornos mentais e de comportamento da CID-10: descrições clínicas e diretrizes diagnósticas. Porto Alegre: Artes Médicas; 1993.

14. Ferreira Filho OF, Turchi MD, Laranjeira R, Castelo A. Perfil sociodemográfico e de padrões de uso entre dependentes de cocaína hospitalizados. Rev Saude Publica. 2003;37(6):751-9.

15. Duailibi LB, Ribeiro M, Laranjeira R. Perfil dos usuários de cocaína e crack no Brasil. Cad Saude Publica. 2008;24(Supl 4):S545-57.

16. Nappo SA, Galduróz JC, Raymundo M, Carlini EA. Changes in cocaine use as viewed by key informants: a qualitative study carried out in 1994 and 1999 in Sao Paulo, Brazil. J Psychoactive Drugs. 2001;33(3):241-53.

17. Ferri CP. Cocaína: padrão de consumo e fatores associados à procura de tratamento [dissertação]. São Paulo: Universidade Federal de São Paulo; 1999.

18. Swift RM, Stout RL. The relationship between craving, anxiety, and other symptoms in opioid withdrawal. J Subst Abuse. 1992;4(1):19-26.

19. Roelofs S. Hyperventilation, anxiety, craving for alcohol: a subacute alcohol withdrawal syndrome. Alcohol. 1985;2(3):501-5.

20. Ito LM. Terapia cognitivo-comportamental para transtornos psiquiátricos. Porto Alegre: Artmed; 1998.

21. Valença AM, Falcão R, Freire RC, Nascimento I, Nascentes R, Zin WA, et al. The relationship between the severity of asthma and comorbidites with anxiety and depressive disorders. Rev Bras Psiquiatr. 2006;28(3):206-8. 